\title{
Multiple Cavernous Angiomas Associated With Psychotic Symptoms: A Case Report
}

\author{
Mohammad Sayadnasiri, ${ }^{1, *}$ and Farbod Fadai ${ }^{1}$ \\ ${ }^{1}$ Psychosis Research Center, University of Social Welfare and Rehabilitation Sciences, Tehran, IR Iran \\ "Corresponding author: Mohammad Sayadnasiri, Psychosis Research Center, University of Social Welfare and Rehabilitation Sciences, Tehran, IR Iran. E-mail: \\ nasiri115@yahoo.com
}

Received 2015 July 09; Revised 2015 August 02; Accepted 2016 September 24.

\begin{abstract}
Introduction: Multiple cerebral cavernous angiomas (CCA) have genetic origin. They are uncommon entity and rarely occurring with psychiatric manifestations.

Case Presentation: A 28- year-old man presented with delusional disorder some months after diagnosis of CCA for which a neurosurgical intervention had been performed. According to clinical and neuroimaging findings, we discuss the possible correlation of CCA with psychotic symptoms of this patient.

Conclusions: Possibly, abnormal brain development secondary to a genetic abnormality or peri-natal insult predisposed patient to two different neuropsychiatric conditions.
\end{abstract}

Keywords: Cavernous Angioma, Cerebral Hemorrhage, Psychosis

\section{Introduction}

Cerebral cavernous angioma (CCA) is a developmental vascular malformation that is composed of small blood cavities surrounded by a single layer of endothelium without muscular tissue or intervening brain parenchyma [1]. Although cerebral hemispheres are the most common location of cavernous angiomas but these vascular malformations can be detected in the spinal cord, retina, and also as hyperkeratotic cutaneous vascular lesions on the skin [1]. In rare cases, CCA is known to be familial, with dominant autosomal transmission [2]. The incidence of CCA is about $0.5 \%$ in the general population and it is estimated that only $20 \%-30 \%$ of affected individuals become symptomatic. CCAs usually manifest with seizure, cerebral hemorrhage, progressive neurologic deficits, and headache [1]. Psychotic symptoms are rarely reported in these patients [2]. We report a rare case of multiple CCAs in the cerebral hemispheres who presented with psychotic features after hemorrhagic complication of the angiomas.

\section{Case Presentation}

A 28-year-old man was admitted to psychiatric ward because of psychotic event, characterized by paranoid delusions and aggressive behavior. He believed that his family was attempting to poison his food and make plan secretly to harm him. Recently, he had low appetite and poor hygiene. He denied a psychological problem and insisted that he had a blood disease. Neurologic examination was non-focal. Family history was negative for psychiatric disorders.

Two years ago, patient presented with serial seizures after 3 - 4 weeks of progressive headache. Brain MRI showed two small ill-defined non-enhancing masses with a mixed intensity core surrounded by a low signal intensity rim on T2 weighted image at right fontal and parietal subcortical areas (Figure 1). Presence of a mixed signal core with a hypointense rim was considered radiological hallmark of cavernous angioma and patient was scheduled for neurosurgical removal of the lesion. Pathologic examination confirmed the diagnosis of cavernous angioma.

\section{Discussion}

Three possible theories can be proposed to explain the association of CCA and psychiatric symptoms in this patient. The first possibility that comes to mind is that the patient's symptoms caused directly by brain injury of CCA hemorrhagic transformation. Given that the main location proposed for brain dysfunction in schizophrenic patients is prefrontal cortex [3], psychotic symptoms of the patient cannot be attributed directly to the site of CCAs in the deep frontal and parietal lobes but according to some studies that proposed a functional interdependency of the 

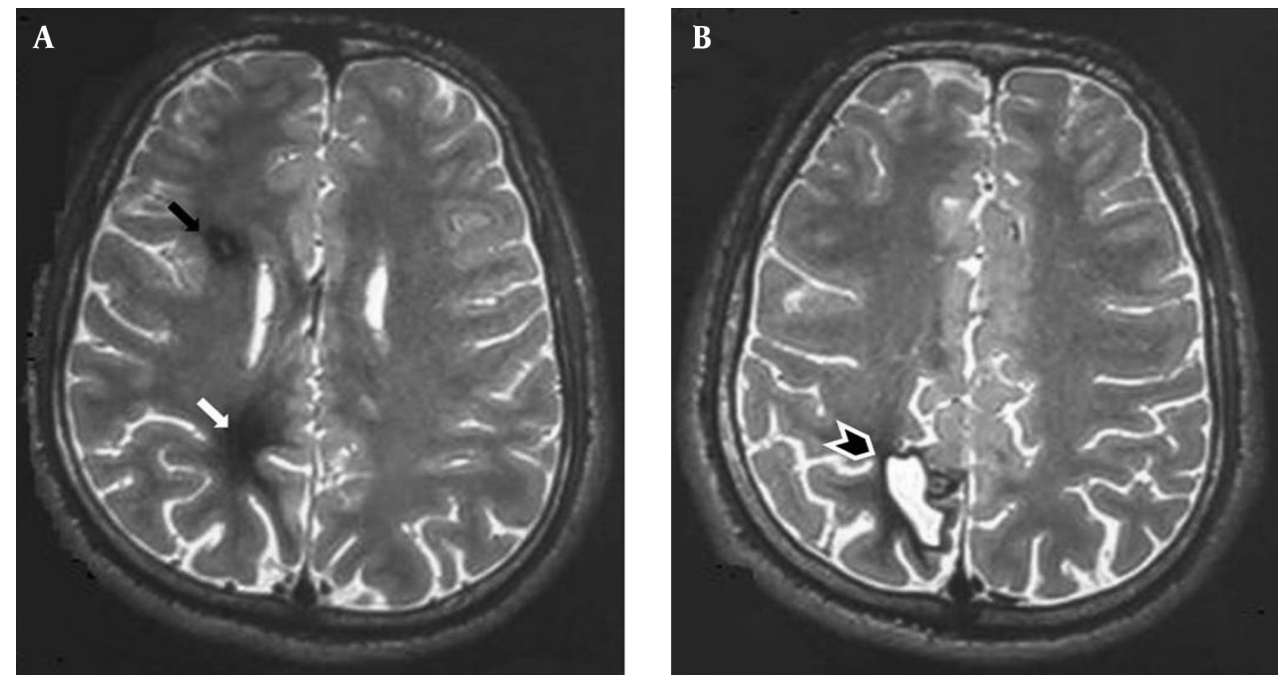

Figure 1. A and B, Axial T2-weighted MR image showing a small mass on the right deep posterior frontal lobe with a mixed intensity core surrounded by a low signal intensity hemosiderin rim (black arrow) in favour of a cavernous angioma and an ill-defined low signal intensity area in right deep posterior parietal lobe indicative of post-surgical gliosis (white arrow) with residual cystic lesion (black arrowhead).

parietal and frontal lobes in psychotic patients [4], a causal relationship may be suggested. In these patients, frontal dysfunction may be compensated by parietal lobe overactivity but in this case, hemorrhagic injury of parietal lobe leading to fronto-parietal network disruption makes this supportive mechanism to work improperly with eventual cognitive performance.

Second possible theory is based on the neurodevelopmental hypothesis of schizophrenia. According to this hypothesis, early insults in peri-natal period and infancy in association with genetic risk factors make the brain susceptible to later development of schizophrenia [5]. However, a long delay is usually present from first insult and symptom appearance; a second insult such as a vascular cerebral injury may be necessary to unfold psychotic symptoms in the patients. On the other hand, a hereditary basis can be sought in most cases of multiple CCAs and dysgenesis of cerebral vascular structures contributes to abnormal brain development of these patients. Therefore, a common pathophysiologic pathway such as genetic susceptibilities or peri-natal insults can be considered as the cause of abnormal brain development and predispose our patient to different neuropsychiatric conditions.

Finally, as the third possibility, CCAs and psychosis can be considered as two co-incidental conditions reflecting two different and irrelevant pathophysiologic processes in this case, although it seems unlikely.

In this case, we prefer the second theory that confirms a neurodevelopmental basis for psychotic symptoms of this patient although expansion and hemorrhagic complica- tion of the CCAs probably triggered off the psychotic symptoms. Such cases with combined organic and psychiatric diseases can open new horizon to theorizing better the pathophysiologic basis of behavioural disease.

\section{References}

1. Revencu N, Vikkula M. Cerebral cavernous malformation: new molecular and clinical insights. J Med Genet. 2006;43(9):716-21. doi: 10.1136/jmg.2006.041079. [PubMed: 16571644].

2. Israeli D, Zohar J. Is multiple cavernoma a developmental defect in schizophrenia?. Am J Psychiatry. 2004;161(5):924. doi: 10.1176/appi.ajp.161.5.924. [PubMed: 15121666].

3. Yildiz M, Borgwardt SJ, Berger GE. Parietal lobes in schizophrenia: do they matter?. Schizophr Res Treatment. 2011;2011:581686. doi: 10.1155/2011/581686. [PubMed: 22937268].

4. Quintana J, Wong T, Ortiz-Portillo E, Kovalik E, Davidson T, Marder SR, et al. Prefrontal-posterior parietal networks in schizophrenia: primary dysfunctions and secondary compensations. Biol Psychiatry. 2003;53(1):12-24. [PubMed: 12513941].

5. Owen MJ, O’Donovan MC, Thapar A, Craddock N. Neurodevelopmental hypothesis of schizophrenia. Br J Psychiatry. 2011;198(3):173-5. doi: 10.1192/bjp.bp.110.084384. [PubMed: 21357874]. 